\title{
Role of the tensor exchange potential in nucleon-nucleus scattering
}

\author{
E. S. Cunningham, ${ }^{1,2,{ }^{*}}$ J. S. Al-Khalili, ${ }^{1}$ and R. C. Johnson ${ }^{1}$ \\ ${ }^{1}$ Department of Physics, University of Surrey, Guildford, Surrey, GU2 7XH, United Kingdom \\ ${ }^{2}$ Royal Observatory Greenwich, Blackheath Avenue, London, SE10 8XJ, United Kingdom
}

(Received 29 June 2011; published 12 October 2011)

\begin{abstract}
We give a microscopic derivation of the spin-spin components of the optical potential for elastic scattering of a nucleon from a target with nonzero spin. A realistic nucleon-nucleon interaction containing direct and exchange terms is used to generate a folded nucleon-nucleus potential whose spin-spin components are treated in distorted-wave Born approximation. We show how folding leads to spin-spin tensor interactions with higher order couplings in the projectile and target spin that have not been explicitly considered before. We place particular emphasis on the polarization transfer coefficient $D_{\mathrm{NN}}$ for $200-\mathrm{MeV}$ protons elastically scattered from ${ }^{10} \mathrm{~B}$, which is rigorously unity in the absence of any spin-spin interactions and for which experimental data exist. We find that deviations of $D_{\mathrm{NN}}$ from unity are particularly sensitive to spin-spin exchange terms arising from the nucleon-nucleon tensor interaction and the nuclear wave functions describing the target one-body density matrix. Unpolarized differential cross sections and vector analyzing powers are found to be very insensitive to these terms.
\end{abstract}

DOI: 10.1103/PhysRevC.84.041601

PACS number(s): 25.40.Cm, 21.30.Fe, 24.70.+s, 24.10.Eq

In order to determine optical potentials for the exotic nuclear species planned to be created in future radioactive beam facilities, it is necessary to establish optical models that better describe interactions for nucleon scattering from nonzero spin targets. Optical potentials are vital for studying the structure of atomic nuclei, but the full spin dependence of the interaction between nuclei with nonzero nuclear spin has not been fully explored. In many scattering experiments spin-spin effects are averaged out because of random projectile or target spin orientations. However, with the next generation of radioactive beam facilities currently under construction there is a vital need for nuclear reaction theory calculations to make predictions of polarization observables relevant to the proposed experiments. In order to do this, it is timely to re-examine the microscopic foundation of the nucleon optical model when the target nucleus has nonzero spin.

Experimental evidence for the dependence of elastic scattering of protons from a nuclear target on the spin of the target has been obtained through the measurement of the polarization transfer coefficient, $D_{\mathrm{NN}}$, by Betker et al. [1]. $D_{\mathrm{NN}}$ (or $D_{\text {yy }}$ as it is sometimes called, where the direction of the $y$ axis is normal to the scattering plane) is related to the nucleon-nucleus scattering amplitude $F$ and the $y$ component $\sigma_{0 y}$, of the projectile nucleon spin operator $\sigma_{0}$, by the following relation [2]:

$$
D_{\mathrm{NN}}=\frac{\operatorname{Tr}\left[F \sigma_{0 y} F^{\dagger} \sigma_{0 y}\right]}{\operatorname{Tr}\left[F F^{\dagger}\right]},
$$

where the trace is taken over the projectile and target spin projections. The theoretical value of this observable is equal to unity unless a term dependent on the orientation of the target spin is present in the potential. It therefore provides a unique test of the spin dependence in the optical potential. Betker et al. measured a significant deviation of $D_{\mathrm{NN}}$ from unity at large

\footnotetext{
*e.cunningham@surrey.ac.uk
}

angles for $200-\mathrm{MeV}$ protons elastically scattering from ${ }^{10} \mathrm{~B}$ at the Indiana University Cyclotron Facility (IUCF). However, their coupled-channel distorted wave impulse approximation (DWIA) calculation of $D_{\mathrm{NN}}$ deviated from unity by only about 5\% at large angles, an order of magnitude smaller than the observed data. Here we address this issue through a microscopic folding model. Due to the $3^{+}$ground state of ${ }^{10} \mathrm{~B}$, spin-spin interactions that have higher order couplings of the target and projectile spins are included.

It should be noted that we do not attempt here to give a complete microscopic description of the proton optical potential. Very extensive fully microscopic calculations of proton scattering by ${ }^{10} \mathrm{~B}$ at $197 \mathrm{MeV}$ including exchange effects have been published in Ref. [3]. These calculations give an excellent account of observables for scattering angles less than 60 degrees, but they fail to give the large deviation of $D_{\mathrm{NN}}$ from unity at large angles observed experimentally [1]. Our approach specifically addresses those components of the optical potential that are known to be responsible for the deviation of $D_{\mathrm{NN}}$ from unity. The remaining components of the optical potential are treated nonmicroscopically.

The effects of tensor forces in nuclei is currently a topic of great interest. In particular, the tensor interaction between nucleons is believed to influence the spin-orbit splitting and shell structure of exotic nuclei [4-7]. It has been found that the two-body tensor force has an important role in shell evolution and the single-particle energy spacing of nuclei far from stability $[8,9]$. It is one of the goals of this work to determine if inclusion of the tensor nucleon-nucleon (NN) interaction in our folding model also has a significant effect on $D_{\mathrm{NN}}$.

Inclusion of terms in the optical potential that depend on the spin operator, $\boldsymbol{I}$, of the target nucleus was first proposed by Feshbach $[10,11]$ and a good review of early work has been written by Sherif [12]. McAbee $[13,14]$ derived generalized spin-spin potentials of the form $U_{k_{l} k}(\boldsymbol{R})=F_{k_{l} k}(R) \boldsymbol{S}_{k_{l} k}$, where $k_{I}$ is the rank of the spin operator constructed from the components of the target spin $\boldsymbol{I}, k$ is the rank of the spherical 
harmonic $Y_{k q}(\hat{\boldsymbol{R}})$, of the unit vector of the projectile-target separation, and $F_{k_{I} k}(R)$ is the form factor. The generalized spin-spin operator $\boldsymbol{S}_{k_{I} k}$ is given by

$$
\boldsymbol{S}_{k_{I} k}=\left[\tau_{1}\left(\boldsymbol{\sigma}_{0}\right) \times \tau_{k_{I}}(\boldsymbol{I})\right]_{k} \cdot Y_{k}(\hat{\boldsymbol{R}}),
$$

where the standard spin operators, $\tau_{1 q_{0}}\left(\boldsymbol{\sigma}_{0}\right)$ and $\tau_{k_{I} q_{I}}(\boldsymbol{I})$, are described in Refs. [15,16]. Microscopic calculations of McAbee's spin-spin potentials were performed using a valence-nucleon model for the target nucleus and an effective $\mathrm{NN}$ interaction. The spin-spin amplitudes were calculated in distorted-wave Born approximation (DWBA). McAbee found that in many cases the spin-spin interactions should not be neglected, especially for high-spin nuclei.

Here we use a two-valence particle model for the target nucleus, thus reducing the $A$-body wave function to a threebody $($ core $+2 N)$ one. We assume the total spins of the two valence nucleons couple together to give the total spin $\boldsymbol{I}$ of the nucleus, and the pair orbit an inert spin-zero core. The spins of the two valence nucleons also couple together to give the maximum allowed rank, $k_{I}$, of the spin operator constructed from the components of the target spin $\boldsymbol{I}$. Therefore, the types of spin-spin interactions allowed are determined not only by the ground-state spin of the target nucleus but also by the orbitals occupied by its valence particles.

In the simplest single-particle shell-model description, the ${ }^{10} \mathrm{~B}$ nucleus is a half-filled $1 p$ shell. In our calculation it is only the nonzero rank target spin tensor components of the one-body density matrix (OBDM) that are relevant. In contrast with other observables, deviations of $D_{\mathrm{NN}}$ from unity are associated only with these components of the OBDM and they have no contributions from closed shells. We generate these components assuming ${ }^{10} \mathrm{~B}$ to be a full $1 s_{1 / 2}$ shell with a proton and neutron hole in the $1 p_{3 / 2}$ shell. These two valence particles couple together to give the spin $I=3$ for the ground-state spin of the nucleus and maximum $k_{I}=3$. Using this simple nuclear wave function is consistent with previous works, which derive microscopically the spin-spin interactions from the two-body interaction between an incident nucleon and a single valence target nucleon $[12,13]$. However, our description of the nucleus will be a limiting factor when comparing the final calculation with the experimental data and improvements are desirable in future work.

In this work the nucleon-nucleon (NN) interaction, $V_{\mathrm{NN}}(0, i)$, between projectile nucleon 0 and target nucleon $i$, is folded over the ground-state wave function, $\Psi_{I, M}^{A}$ of the target nucleus to derive terms in the optical potential dependent on target spin $I$ with projection $M$. The folded potential between the projectile and target nucleus, $A$, is split into two components,

$$
\begin{aligned}
\left(\Psi_{I, M_{f}}^{A}|V| \Psi_{I, M_{i}}^{A}\right)= & \left\langle\Phi_{\text {core }}\left|\sum_{i=3}^{A} V_{\mathrm{NN}}(0, i)\right| \Phi_{\text {core }}\right\rangle \delta_{M_{i}, M_{f}} \\
& +\left\langle\Psi_{I, M_{f}}\left|\sum_{i=1,2} V_{\mathrm{NN}}(0, i)\right| \Psi_{I, M_{i}}\right\rangle,
\end{aligned}
$$

with the core and valence particle wave functions denoted as $\Phi_{\text {core }}$ and $\Psi_{I, M}$ respectively. The first matrix element on the right-hand side denotes the interaction between the projectile and spin-zero core. This interaction, and the nonspin-spin components of the second term in Eq. (3) have central and spin-orbit parts that are included through a target spin-independent phenomenological optical potential, taken from Ref. [1] for $200-\mathrm{MeV}$ protons scattering from ${ }^{10} \mathrm{~B}$. This potential was fitted to differential cross section and analyzing power measurements, which are insensitive to the effects of spin-spin interactions. As the effect of all the spin-spin interactions discussed in this work on the calculation of the cross section and vector analyzing power is negligible they will not be plotted here. The second term in Eq. (3) is the interaction between the projectile and valence nucleons. It is from this term that the target spin-dependent spin-spin potentials are derived. While DWBA was used to calculate the scattering amplitude from the spin-spin interactions, the scattering amplitude from the nucleon-nucleus interactions (not including spin-spin) was calculated exactly.

The two-body effective NN interaction $V_{\mathrm{NN}}(a, b)$ between nucleon $a$ and nucleon $b$, with separation, $r$, can be written as

$$
\begin{aligned}
V_{\mathrm{NN}}(a, b)= & v_{\mathrm{NN}}^{\mathrm{cen}}(r)+v_{\mathrm{NN}}^{\sigma \sigma}(r) \sigma_{a} \cdot \boldsymbol{\sigma}_{b} \\
& +v_{\mathrm{NN}}^{\mathrm{ls}}(r) \boldsymbol{\ell}_{a b} \cdot\left(\sigma_{a}+\sigma_{b}\right)+v_{\mathrm{NN}}^{\mathrm{tr}}(r) \boldsymbol{S}_{a b},
\end{aligned}
$$

where the relative angular momentum is

$$
\boldsymbol{\ell}_{a b}=(2 \hbar)^{-1} \boldsymbol{r} \times\left(\boldsymbol{p}_{a}-\boldsymbol{p}_{b}\right)
$$

and $\boldsymbol{p}$ is a nucleon momentum. The NN tensor operator is

$$
\boldsymbol{S}_{a b}=3\left(\boldsymbol{\sigma}_{a} \cdot \hat{\boldsymbol{r}}\right)\left(\boldsymbol{\sigma}_{b} \cdot \hat{\boldsymbol{r}}\right)-\boldsymbol{\sigma}_{a} \cdot \boldsymbol{\sigma}_{b},
$$

and $v_{\mathrm{NN}}^{\mathrm{cen}}, v_{\mathrm{NN}}^{\sigma \sigma}, v_{\mathrm{NN}}^{\mathrm{ls}}$, and $v_{\mathrm{NN}}^{\mathrm{tr}}$ are the radial form factors for central spin-independent, central spin-spin, spin-orbit, and tensor interactions. This work focuses on spin-spin interactions from the folding of the central spin-spin and tensor terms in the $\mathrm{NN}$ interaction. These give all the local spin-spin interactions that can be formed from the coupling of $\boldsymbol{\sigma}_{0}, \boldsymbol{I}$, and $\boldsymbol{R}$ given in Eq. (2) for the generalized spin-spin operator. However, spin-spin potentials derived from the folding of the spin-orbit term were also proposed by Feshbach [10]. Some studies of the first-order $\boldsymbol{I} \cdot \boldsymbol{l}$ term have been performed, where $\boldsymbol{l}$ is the relative orbital angular momentum of the projectile and target [17]. This term by itself does not give a deviation of $D_{\mathrm{NN}}$ from unity. If the scattering amplitude is independent of the incident proton spin Eq. (1) shows that $D_{\mathrm{NN}}=1$. The folding of the central spin-independent term in Eq. (4) does not result in spin-spin interactions.

We use a realistic free-space $\mathrm{NN}$ interaction that is an improved version of the Bonn-B potential. The parameters of the original Bonn-B potential have been adjusted to bring the predictions into better agreement with subsequent high-precision measurements of NN scattering and phaseshift analysis (see references in [18]). We use an effective interaction that is a superposition of Yukawa terms fitted to a free, complex $t$ matrix and are calculated for an incident proton laboratory energy of $200 \mathrm{MeV}$, as described in Ref. [18].

For the case of nucleon-nucleus scattering, the total nuclear wave function must be antisymmetric under the interchange of nucleons between the projectile and target. For nucleonnucleus scattering it is reasonable, when considering exchange 
contributions arising from antisymmetrization, to use a singlenucleon knock-on exchange (SNKE) correction, where a target nucleon is ejected and replaced by the projectile nucleon after their interaction [19]. To formally include knock-on exchange in a folding model, the effective NN interaction potential term, $v_{p t}(r)$, between projectile nucleon $p$ and target nucleon $t$, can be replaced by

$$
v_{p t}(r) \rightarrow\left(1-P_{p t}\right) v_{p t}(r),
$$

where $P_{p t}$ is the operator that exchanges all the coordinates of the two nucleons [20,21]. The exchange operator can be written in terms of the spin and isospin operators $P^{\sigma}$ and $P^{\tau}$, which exchange the spin and isospin coordinates, respectively,

$$
P_{p t}=P_{p t}^{\sigma} P_{p t}^{\tau} P_{p t}^{r}
$$

where $P_{p t}^{r}$ exchanges the spatial coordinates of the two nucleons. The NN potential, $v_{p t}(r)$ can now be written as

$$
\begin{aligned}
v_{p t}(r) & \rightarrow\left(1-P_{p t}\right) v_{p t}(r), \\
& \rightarrow v_{p t}(r)+\hat{v}_{p t}(r) P_{p t}^{r},
\end{aligned}
$$

where $\hat{v}_{p t}(r)=-P_{p t}^{\sigma} P_{p t}^{\tau} v_{p t}(r)$ and has a spin-isospin decomposition that is the same as $v_{p t}(r)$, except that the sign for the odd-state terms is changed.

The effect of exchange terms on spin-spin interactions derived from folding models has yet to be fully investigated. Early folding models for spin-spin interactions used effective interactions, which were mainly phenomenological, so exchange was included crudely [12]. However, explicit exchange effects were not incorporated, although they were discussed [22]. Petrovich [23] and McAbee [13] used SNKE to approximate exchange for the $v_{\mathrm{NN}}^{\sigma \sigma}(r) \sigma_{a} \cdot \boldsymbol{\sigma}_{b}$ term in their effective NN interaction. Petrovich also approximated the spin-orbit exchange terms using the odd state spin-orbit components alone, but both works neglected exchange effects in the tensor terms in their final calculations. Our work aims to include SNKE terms consistently for both the central $v_{\mathrm{NN}}^{\sigma \sigma}(r) \boldsymbol{\sigma}_{a} \cdot \boldsymbol{\sigma}_{b}$ and tensor $v_{\mathrm{NN}}^{\mathrm{tr}}(r) \boldsymbol{S}_{a b}$ terms in the effective NN interaction and investigate their contributions to the spin-spin interactions.

In order to avoid the evaluation of exact complicated exchange amplitudes, the use of zero-range pseudopotentials has been adopted for treating exchange terms in folding models [24-26]. In the simplest of these approximate treatments the exchange pseudopotentials are derived to yield the same Born amplitudes as their equivalent exchange terms [19,27]. The resulting zero-range effective exchange interaction can then be used to evaluate the scattering amplitude more readily than for the full exchange terms.

The zero-range pseudopotential used to approximate the central spin-spin exchange term is given by

$$
\hat{v}_{\mathrm{NN}}^{\sigma \sigma}(r) \boldsymbol{\sigma}_{a} \cdot \boldsymbol{\sigma}_{b} \quad P_{a b}^{r} \rightarrow \hat{J}_{\sigma \sigma}(Q) \delta(\boldsymbol{r}) \boldsymbol{\sigma}_{a} \cdot \boldsymbol{\sigma}_{b} P_{a b}^{r},
$$

where the strength $\hat{J}_{\sigma \sigma}(Q)$ is the Fourier transform of $\hat{v}_{\mathrm{NN}}^{\sigma \sigma}(r) P_{a b}^{r}$,

$$
\hat{J}_{\sigma \sigma}(Q)=4 \pi \int_{0}^{\infty} j_{0}(Q r) \hat{v}_{\mathrm{NN}}^{\sigma \sigma}(r) r^{2} d r
$$

in terms of the spherical Bessel function, $j_{0}(Q r)$, where $Q$ is the sum of initial, $\boldsymbol{k}$, and final, $\boldsymbol{k}^{\prime}$, wave vectors of either particle in the center of mass system,

$$
Q=\boldsymbol{k}^{\prime}+\boldsymbol{k}
$$

This pseudopotential for the central exchange contribution to the NN interaction has been used by several authors and has been shown to be reasonably accurate for nucleon scattering at energies above $100 \mathrm{MeV}$ (see references in [19-21]).

In previous work on spin-spin interactions, the contribution of the tensor exchange term in the $\mathrm{NN}$ interaction has been neglected due to the lack of a pseudopotential approach for the noncentral tensor potential [14]. In this work the tensor exchange is included using the pseudopotential approximation in a process analogous to that of the central spin-spin exchange. The pseudopotential used to approximate the tensor term in this work is

$\hat{v}_{\mathrm{NN}}^{\mathrm{tr}}(r) \boldsymbol{S}_{a b} P_{a b}^{r} \rightarrow \hat{J}_{\mathrm{tr}}(Q)\left[\hat{\boldsymbol{S}}_{a b}\left(\boldsymbol{\sigma}_{a}, \boldsymbol{\sigma}_{b},-l \nabla_{\boldsymbol{r}}\right) \delta(\boldsymbol{r})\right] P_{a b}^{r}$,

where the strength $\hat{J}_{\mathrm{tr}}(Q)$ is calculated using the Fourier transform

$$
\hat{J}_{\mathrm{tr}}(Q)=-\frac{4 \pi}{Q^{2}} \int_{0}^{\infty} j_{2}(Q r) \hat{v}_{\mathrm{NN}}^{\mathrm{tr}}(r) r^{2} d r .
$$

The operator $\hat{\boldsymbol{S}}_{a b}$ in Eq. (14) is

$$
\begin{aligned}
\hat{\boldsymbol{S}}_{a b}\left(\boldsymbol{\sigma}_{a}, \boldsymbol{\sigma}_{b},-\imath \nabla_{\boldsymbol{r}}\right)= & 3\left(\boldsymbol{\sigma}_{a} \cdot\left[-\imath \nabla_{\boldsymbol{r}}\right]\right)\left(\boldsymbol{\sigma}_{b} \cdot\left[-\imath \nabla_{\boldsymbol{r}}\right]\right) \\
& -\left[-\imath \nabla_{\boldsymbol{r}}\right]^{2} \boldsymbol{\sigma}_{a} \cdot \boldsymbol{\sigma}_{b} .
\end{aligned}
$$

The form of the tensor exchange term given in Eq. (14) is nonlocal, which significantly complicates the calculation of the folded potential and observables. This is one of the reasons these terms have been neglected in the past [13].

The exchange terms change the dependence of the Born amplitudes from the momentum transfer $\boldsymbol{q}=\boldsymbol{k}^{\prime}-\boldsymbol{k}$, to $\boldsymbol{Q}=$ $\boldsymbol{k}^{\prime}+\boldsymbol{k}$, through the action of the $P_{a b}^{r}$ exchange operator, which interchanges the spatial coordinates of the two nucleons. In order to evaluate the Born amplitudes for the exchange terms, a value must be chosen for the magnitude of $\boldsymbol{Q}$. The simplest choice is to take this to be the center-of-mass wave number for the incident particle: $Q=k_{\mathrm{cm}}[18,19]$. This choice is reasonable for scattering in the forward direction. However, the measured deviation of $D_{\mathrm{NN}}$ from unity is most significant at large scattering angles. Taking the value of $Q$ associated with a scattering angle of $\theta=0^{\circ}$ would therefore have limited validity. In order to perform an improved calculation of these exchange terms at large scattering angles the approximation $Q=k \cos (\theta / 2)$ is used here [18].

Some of the folded spin-spin potentials derived from the tensor exchange term in the NN interaction have the same form as the generalized (local) spin-spin operator given in Eq. (2). However, the folding of this exchange term also results in spin-spin potentials with nonlocal operators of the form

$$
\sum_{\epsilon}\left[Y_{k}(\hat{\boldsymbol{R}}) \times \tau_{k_{I}}(\boldsymbol{I})\right]_{E \epsilon}\left[\tau_{1}\left(\boldsymbol{\sigma}_{0}\right) \times \mathscr{Y}_{k^{\prime}}\left(-\imath \nabla_{\boldsymbol{R}}\right)\right]_{E-\epsilon},
$$




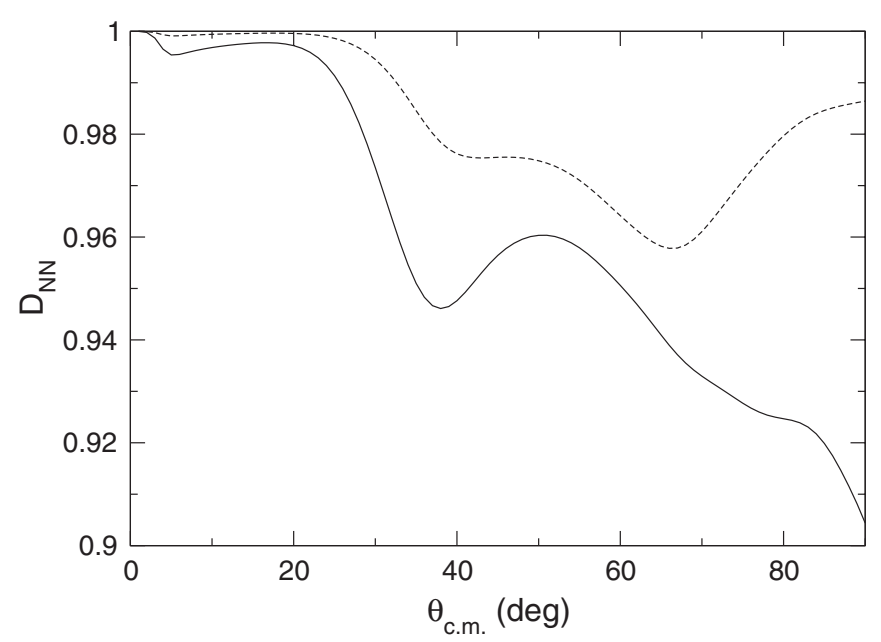

FIG. 1. Calculation of $D_{\mathrm{NN}}$ including all spin-spin interactions (solid line), and without tensor exchange contributions (dashed line), for elastic proton scattering from ${ }^{10} \mathrm{~B}$.

with $k^{\prime}=1$ and 2 , and

$$
\mathscr{Y}_{k^{\prime} q^{\prime}}(\boldsymbol{A})=A^{2} Y_{k^{\prime} q^{\prime}}(\hat{\boldsymbol{A}}) .
$$

We have modified the calculation of the DWBA scattering amplitude to include nonlocal spin-spin operators to enable us to examine the effect of tensor exchange on spin-spin terms in the optical potential.

The result for the polarization transfer coefficient, $D_{\mathrm{NN}}$, for all the spin-spin potentials derived here is shown in Fig. 1 by the solid line. The dashed curve shows the same calculation with no tensor exchange contributions. The difference between these two curves is most significant at large angles where the deviation of $D_{\mathrm{NN}}$ from unity was measured to be greatest [1]. Without tensor exchange the deviation of $D_{\mathrm{NN}}$ from unity is less than $5 \%$ and is comparable with the coupled-channel DWIA calculation carried out in Betker et al. [1].

The single-particle wave functions for the valence proton and neutron in the $1 p_{3 / 2}$ shell of the ${ }^{10} \mathrm{~B}$ nucleus are calculated from a Woods-Saxon (WS) potential. The WS parameters $R_{\mathrm{WS}}=(0.88 \pm 0.13)(A-1)^{1 / 3}$ fm for the well radius and $a=0.81 \pm 0.08$ fm for the diffuseness are taken from Ref. [28], who obtain the $1 p_{3 / 2}$ wave function from the transform of the electron scattering form factor of the $1.740 \mathrm{MeV}$ excited state in ${ }^{10} \mathrm{~B}$. To examine the sensitivity of $D_{\mathrm{NN}}$ to the WS parameters, a range of values for $R_{\mathrm{WS}}$ and $a$, determined by the uncertainties quoted in Ref. [28] were investigated. The corresponding angular distributions of $D_{\mathrm{NN}}$ are shows by the shaded area in Fig. 2. The solid line in Fig. 2 shows the calculation of $D_{\mathrm{NN}}$ using the WS parameters $R_{\mathrm{WS}}=0.88(A-1)^{1 / 3} \mathrm{fm}$ and $a=0.81 \mathrm{fm}$ to calculate the single-particle wave function and is the same as the solid line in Fig. 1. The circular points denote the experimentally measured values of $D_{\mathrm{NN}}$ [1]. The large difference between the curves depends not only on the single-particle wave function but also on its first and second derivatives through the tensor exchange terms. Therefore, the inclusion of the tensor

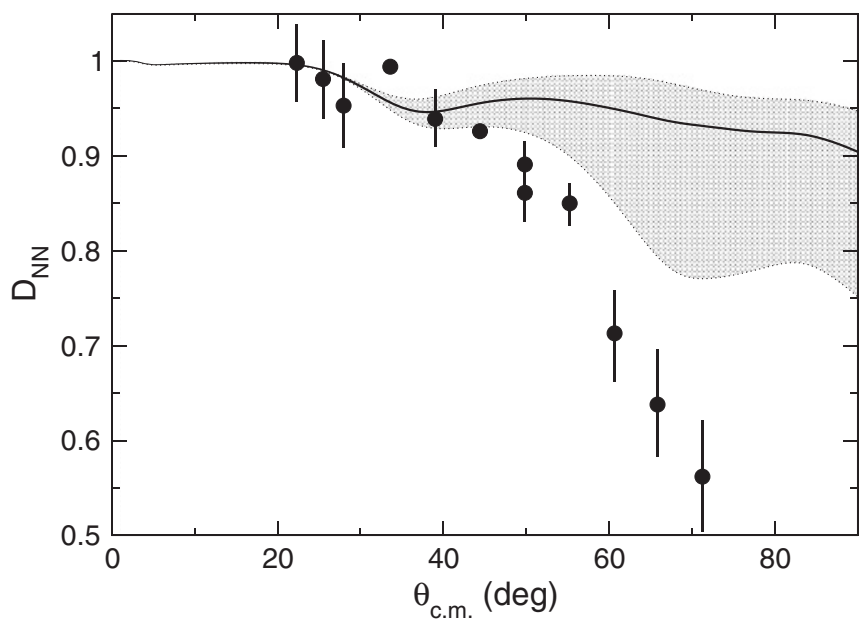

FIG. 2. Calculation of $D_{\mathrm{NN}}$, for elastic proton scattering from ${ }^{10} \mathrm{~B}$ (solid line) compared to the measurement (symbols) of $D_{\mathrm{NN}}$ from Ref. [1]. The grey area shows the dependence of the calculation on the uncertainties in the Woods-Saxon parameters from Ref. [28] used to calculate the single-particle wave function.

exchange contributions to spin-spin interactions not only leads to the significant deviation of $D_{\mathrm{NN}}$ from unity shown in Fig. 1, but also makes this polarization observable very sensitive to changes in the shape of the wave function. This sensitivity may make $D_{\mathrm{NN}}$ a potentially useful probe of nuclear structure.

While this polarization transfer coefficient was identified by Stamp to be the simplest way of verifying experimentally the existence of spin-spin interactions [29], we have also determined that the dependence of $D_{\mathrm{NN}}$ on the strength of the spin-spin interactions is second order [30,31]. A better approach may be to study first-order effects of spin-spin interactions using another observable. Since $D_{\mathrm{NN}}$ is only dependent on the polarization of the projectile nucleon, an observable that is dependent on the polarization of the target would lead to a first-order dependence on the strength of the spin-spin interactions. Such an observable would be a more sensitive probe of spin-spin interactions.

In summary, we have found that the spin-spin interactions derived from this model cause a significant deviation of $D_{\mathrm{NN}}$ from unity and this deviation is very sensitive to nuclear structure. We find that the role of the tensor exchange contribution is particularly important at large angles where the deviation of $D_{\mathrm{NN}}$ from unity is measured to be greatest. However, while our calculations do lead to a significant deviation of $D_{\mathrm{NN}}$ from unity, they still do not match the experimental data from Ref. [1] at large angles. The calculation of $D_{\mathrm{NN}}$ has been shown to be particularly sensitive to the occupation of different orbitals and their radial wave functions. This sensitivity means polarization observables could be used as a probe of nuclear structure.

The authors would like to thank Ed Stephenson for alerting us to this problem in the first place and for many helpful discussions. 
[1] A. C. Betker et al. Phys. Rev. C 71, 064607 (2005).

[2] E. Bleszynski, M. Bleszynski, and C. A. Whitten Jr., Phys. Rev. C 26, 2063 (1982).

[3] K. Amos, S. Karataglidis, and Y. J. Kim, Nucl. Phys. A 836, 59 (2010).

[4] B. A. Brown, T. Duguet, T. Otsuka, D. Abe, and T. Suzuki, Phys. Rev. C 74, 061303 (2006).

[5] D. M. Brink and Fl. Stancu, Phys. Rev. C 75, 064311 (2007).

[6] M. Zalewski, W. Satuła, J. Dobaczewski, P. Olbratowski, M. Rafalski, T. R. Werner, and R. A. Wyss, Eur. Phys. J. A 42, 577 (2009).

[7] E. B. Suckling and P. D. Stevenson, Europhys. Lett. 90, 12001 (2010).

[8] T. Otsuka, R. Fujimoto, Y. Utsuno, B. A. Brown, M. Honma, and T. Mizusaki, Phys. Rev. Lett. 87, 082502 (2001).

[9] T. Otsuka, T. Suzuki, R. Fujimoto, H. Grawe, and Y. Akaishi, Phys. Rev. Lett. 95, 232502 (2005).

[10] H. Feshbach, Annu. Rev. Nucl. Sci. 8, 49 (1958).

[11] H. Feshbach, in Nuclear Spectroscopy, Part B, edited by F. Ajzenberg-Selove (Academic Press, New York, 1960), p. 1033.

[12] H. S. Sherif, in Proceedings of the 4th International Symposium on Polarization Phenomena in Nuclear Reactions, Zurich., edited by W. Gruebler and V. Konig (Birkhauser, Basel, 1976), p. 189.

[13] T. L. McAbee, W. J. Thompson, and H. Ohnishi, Nucl. Phys. A 509, 39 (1990).

[14] T. L. McAbee, Ph.D. thesis, University of North Carolina, 1986 (unpublished).
[15] D. J. Hooton and R. C. Johnson, Nucl. Phys. A 175, 583 (1971).

[16] D. M. Brink and G. R. Satchler, Angular Momentum, 3rd ed. (Oxford University Press, Oxford, 1993).

[17] R. C. Barrett and D. F. Jackson, Nuclear Sizes and Shapes (Clarendon Press, Oxford, 1977), p. 203.

[18] F. Sammarruca, E. J. Stephenson, and K. Jiang, Phys. Rev. C 60, 064610 (1999).

[19] G. R. Satchler, Direct Nuclear Reactions (Oxford University Press, New York, 1983).

[20] G. R. Satchler and W. G. Love, Phys. Rep. 55, 183 (1979).

[21] M. E. Brandan and G. R. Satchler, Phys. Rep. 285, 143 (1997).

[22] G. R. Satchler, Part. and Nuclei 1, 397 (1971).

[23] F. Petrovich, R. J. Philpott, A. W. Carpenter, and J. A. Carr, Nucl. Phys. A 425, 609 (1984).

[24] F. Petrovich, H. McManus, V. A. Madsen, and J. Atkinson, Phys. Rev. Lett. 22, 895 (1969).

[25] M. Golin, F. Petrovich, and D. Robson, Phys. Lett. B 64, 253 (1976).

[26] G. R. Satchler and W. G. Love, Phys. Lett. B 65, 415 (1976).

[27] M. LeMere and Y. C. Tang, Phys. Rev. C 19, 391 (1979).

[28] A. Cichocki, J. Dubach, R. S. Hicks, G. A. Peterson, C. W. de Jager, H. de Vries, N. Kalantar-Nayestanaki, and T. Sato, Phys. Rev. C 51, 2406 (1995).

[29] A. P. Stamp, Phys. Rev. 153, 1052 (1967).

[30] E. S. Cunningham, Ph.D. thesis, University of Surrey, 2010 (unpublished).

[31] E. S. Cunningham, J. S. Al-Khalili, and R. C. Johnson (in preparation). 